\title{
Unification of probability theory on time scales
}

Ünal Ufuktepe*

To my wife

"Correspondence:

unal.ufuktepe@ieu.edu.tr Department of Mathematics, Izmir

University of Economics, Sakarya

Cad. No:156 Balçova, İzmir, Turkey

\begin{abstract}
The theory of time scales was introduced by Stefan Hilger in his PhD thesis in 1988 in order to unify continuous and discrete analysis. Probability is a discipline in which appears to be many applications of time scales. Time scales approach to probability theory unifies the standard discrete and continuous random variables. We give some basic random variables on the time scales. We define the distribution functions on time scales and show their properties.
\end{abstract}

MSC: 46N30; 60B05

Keywords: probability; time scales

\section{Introduction}

A time scale or a measure chain was introduced by Stefan Hilger in his PhD thesis in 1988 in order to unify continuous and discrete analysis [1]. We refer the reader to the textbooks [2-4].

Time scale calculus has received a lot of attention [1,5-7]. In recent years there have been many research activities about applications of time scales. Probability theory is an ideal discipline for applications of time scales since random variables and distributions functions can be described with either discrete or continuous cases.

We give a brief introduction to measure theory on time scales introduced by Guseinov [8] in Section 2. We give the discussion of our original probability results in Section 3. In Section 4 we study the discrete random variables, i.e., binomial, Poisson, geometric, and negative binomial random variables on a discrete time scale $h \mathbb{N}$. In Section 4.5 we define uniform random variables on the time scale, and we give the definition of Gaussian bell in Section 5 .

\section{Measure on time scales}

The Riemann $\Delta$ integral has been introduced by Guseinov in [9], the $\Delta$ measure and the Lebesgue $\Delta$ integral were introduced by Guseinov in [8] and studied by Cabada [10], Ufuktepe and Deniz in [11], and Rzezuchowski in [12]. In this section we set out basic concepts of $\Delta$ and $\nabla$ measures.

Let $\mathbb{T}$ be a time scale, $a<b$ be points in $\mathbb{T}$, and $[a, b)$ be a half-closed bounded interval in $\mathbb{T}, \sigma$ and $\rho$ be the forward and backward jump operators respectively on $\mathbb{T}$. Let

$$
\Im_{1}=\left\{\left[a^{\prime}, b^{\prime}\right) \cap \mathbb{T}: a^{\prime}, b^{\prime} \in \mathbb{T}, a^{\prime} \leq b^{\prime}\right\}
$$

(c) 2012 Ufuktepe; licensee Springer. This is an Open Access article distributed under the terms of the Creative Commons Attribution License (http://creativecommons.org/licenses/by/2.0), which permits unrestricted use, distribution, and reproduction in any medium, provided the original work is properly cited. 
be the family of all left closed and right open intervals of $\mathbb{T}$. Then $\Im_{1}$ is a semiring. Here $\left[a^{\prime}, a^{\prime}\right)=\emptyset \cdot m_{1}: \Im_{1} \rightarrow[0, \infty]$ is a set function which assigns to each interval its length: $m_{1}\left(\left[a^{\prime}, b^{\prime}\right)\right)=b^{\prime}-a^{\prime}$. So, if $\left\{I_{n}\right\}$ is a sequence of disjoint intervals in $\Im_{1}$, then $m_{1}\left(\bigcup I_{n}\right)=$ $\sum m_{1}\left(I_{n}\right)$.

Let $E \subset \mathbb{T}$. By the Carathéodory extension, the outer measure of $E$ is

$$
m_{1}^{*}(E)=\inf _{E \subset \bigcup_{n} I_{n}} \sum m_{1}\left(I_{n}\right)
$$

where $I_{n} \in \Im_{1}$. If there is no such covering of $E$, then $m_{1}^{*}(E)=\infty$.

Definition 2.1 A set $E \subset \mathbb{T}$ is said to be $\Delta$-measurable if for each set $A$,

$$
m_{1}^{*}(A)=m_{1}^{*}(A \cap E)+m_{1}^{*}\left(A \cap E^{c}\right)
$$

where $E^{c}=\mathbb{T}-E$.

Since we always have $m_{1}^{\prime \prime}(A) \leq m_{1}^{\prime \prime}(A \cap E)+m_{1}^{*}\left(A \cap E^{c}\right)$, we see that $E$ is $\Delta$-measurable iff for each $A$ we have $m_{1}^{*}(A) \geq m_{1}^{*}(A \cap E)+m_{1}^{*}\left(A \cap E^{c}\right)$.

If $E$ is $\Delta$-measurable, then $E^{c}$ is also $\Delta$-measurable. Clearly, $\varnothing$ and $\mathbb{T}$ are $\Delta$-measurable.

Lemma 2.2 If $E_{1}$ and $E_{2}$ are $\Delta$-measurable, so is $E_{1} \cup E_{2}$.

Let $\mathfrak{M}\left(m_{1}^{*}\right)=\{E \subset \mathbb{T}: E$ is $\Delta$-measurable $\}$ be a family of $\Delta$-measurable sets.

Corollary $2.3 \mathfrak{M}\left(m_{1}^{*}\right)$ is a $\sigma$ algebra.

Definition 2.4 The restriction of $m_{1}^{*}$ to $\mathfrak{M}\left(m_{1}^{*}\right)$ is called the Lebesgue $\Delta$-measure and denoted by $\mu_{\Delta}$.

So, $m_{1}^{*}(E)=\mu_{\Delta}(E)$ if $E \in \mathfrak{M}\left(m_{1}^{*}\right)$. Similarly, if we take

$$
\mathfrak{F}_{2}=\left\{\left(a^{\prime}, b^{\prime}\right]: a^{\prime}, b^{\prime} \in \mathbb{T}, a^{\prime} \leq b^{\prime}\right\},
$$

where $\left(a^{\prime}, a^{\prime}\right]$ is understood as an empty set, then $m_{2}: \mathfrak{F}_{2} \rightarrow[0, \infty]$ such that $m_{2}\left(\left(a^{\prime}, b^{\prime}\right]\right)=$ $b^{\prime}-a^{\prime}$ is a countably additive measure. Then $\mathfrak{M}\left(m_{2}^{*}\right)$ is the set of $\nabla$-measurable sets and $\mu_{\nabla}$ is the Lebesgue $\nabla$-measure on $\mathbb{T}$.

Proposition 2.5 Let $\left\{E_{n}\right\}$ be an infinite decreasing sequence of $\Delta$-measurable sets, that is, a sequence $E_{1} \supset E_{2} \supset \cdots \supset E_{n} \supset \cdots, E_{i} \in \mathfrak{F}_{1}$ for each $i, \bigcap E_{i} \in \mathfrak{F}_{1}$ and $m_{1}^{\prime \prime}\left(E_{1}\right)<\infty$. Then

$$
m_{1}^{*}\left(\bigcap_{n=1}^{\infty} E_{i}\right)=\lim _{n \rightarrow \infty} m_{1}^{*}\left(E_{n}\right) .
$$

Proof [13].

Proposition 2.6 (Properties of $m_{1}^{*}$ )

(i) $m_{1}^{*}(\varnothing)=0$;

(ii) If $E \subset F$, then $m_{1}^{*}(E) \leq m_{1}^{*}(F)$; 
(iii) If $\left\{E_{n}\right\}_{n=1}^{\infty}$ is a sequence of elements of $\mathfrak{F}_{1}$, then

$$
m_{1}^{*}\left(\bigcup_{n=1}^{\infty} E_{n}\right) \leq \sum_{n=1}^{\infty} m_{1}^{*}\left(E_{n}\right)
$$

Proof Similar to the proof in [13].

Theorem 2.7 For each $t_{0} \in \mathbb{T}-\{\max \mathbb{T}\}$, the single point set $\left\{t_{0}\right\}$ is $\Delta$-measurable and its $\Delta$-measure is given by $\mu_{\Delta}\left(\left\{t_{0}\right\}\right)=\sigma\left(t_{0}\right)-t_{0}$.

Proof Case 1 . Let $t_{0}$ be right scattered. Then $\left\{t_{0}\right\}=\left[t_{0}, \sigma\left(t_{0}\right)\right) \in \mathfrak{F}_{1}$. So, $\left\{t_{0}\right\}$ is $\Delta$-measurable and $\mu_{\Delta}\left(\left\{t_{0}\right\}\right)=\sigma\left(t_{0}\right)-t_{0}$.

Case 2. Let $t_{0}$ be right dense. Then there exists a decreasing sequence $\left\{t_{k}\right\}$ of points of $\mathbb{T}$ such that $t_{0} \leq t_{k}$ and $t_{k} \downarrow t_{0}$. Since $\left\{t_{0}\right\}=\bigcap_{k=1}^{\infty}\left[t_{0}, t_{k}\right) \in \mathfrak{F}_{1}$. Therefore, $\left\{t_{0}\right\}$ is $\Delta$-measurable. By Proposition 2.5,

$$
\begin{aligned}
\mu_{\Delta}\left(\left\{t_{0}\right\}\right) & =\mu_{\Delta}\left(\bigcap_{k=1}^{\infty}\left[t_{0}, t_{k}\right)\right) \\
& =\lim _{n \rightarrow \infty} \mu_{\Delta}\left(\left[t_{0}, t_{n}\right)\right) \\
& =\lim _{n \rightarrow \infty} t_{n}-t_{0}=0,
\end{aligned}
$$

which is the desired result since $t_{0}$ is right dense.

Every kind of interval can be obtained from an interval of the form $[a, b)$ by adding or subtracting the end points $a$ and $b$. Then each interval of $\mathbb{T}$ is $\Delta$-measurable.

Theorem 2.8 If $a, b \in \mathbb{T}$ and $a \leq b$, then

(i) $\mu_{\Delta}([a, b))=b-a$;

(ii) $\mu_{\Delta}((a, b))=b-\sigma(a)$;

(iii) If $a, b \in \mathbb{T}-\max \mathbb{T}$, then $\mu_{\Delta}((a, b])=\sigma(b)-\sigma(a)$ and $\mu_{\Delta}([a, b])=\sigma(b)-a$.

Proof $[8]$.

Theorem 2.9 For each $t_{0} \in \mathbb{T}-\{\min \mathbb{T}\}$, the $\nabla$-measure of the single point set $\left\{t_{0}\right\}$ is given by $\mu_{\nabla}\left(\left\{t_{0}\right\}\right)=t_{0}-\rho\left(t_{0}\right)$.

Proof Similar to $\mu_{\Delta}$ case.

Theorem 2.10 If $a, b \in \mathbb{T}$ and $a \leq b$, then

(i) $\mu_{\nabla}((a, b])=b-a$;

(ii) $\mu_{\nabla}((a, b))=\rho(b)-a$;

(iii) If $a, b \in \mathbb{T}-\min \mathbb{T}$, then $\mu_{\nabla}([a, b))=\rho(b)-\rho(a)$ and $\mu_{\nabla}([a, b])=b-\rho(a)$.

Proof The equalities can be obtained by the same technique with $\mu_{\Delta}$ case.

Lemma 2.11 $\lambda^{*}(E) \leq m_{1}^{*}(E)$, where $\lambda^{*}(E)$ is the outer measure of $E$. 


\section{Example}

(i) $\mathbb{T}=\left\{0, \frac{1}{3}, \frac{2}{3}, 1, \frac{4}{3}, \ldots\right\}$ and $A=\{0,1,3\}$, then $\mu_{\Delta}(A)=1$.

(ii) Let $\mathbb{T}=\{0,1,2,3,4, \ldots\}$ and $A=\{1,3,5,6\}$, then $\mu_{\Delta}(A)=4$.

\section{Probability on time scales}

Let $\mathbb{T}$ be any time scale (which may be finite or infinite) and $A \subset \mathbb{T}$, then $A$ can be written as

$$
A=\bigcup_{i=1}^{m}\left[a_{i}, b_{i}\right]_{T} \cup\left\{t_{1}, t_{2}, \ldots, t_{n}\right\},
$$

where $m$ and $n$ are nonnegative integers which may be finite or infinite, $a_{i}$ is right dense, $b_{i}$ is left dense for all $i=1,2, \ldots, m$, all interior points of $\left[a_{i}, b_{i}\right]_{T}$ are dense points, and $t_{i}$ is an isolated point for $j=1,2, \ldots, n$. So, the Lebesgue $\Delta$-measure of $A \neq \emptyset$ is

$$
\mu_{\Delta}(A)=\sum_{i=1}^{m}\left(\sigma\left(b_{i}\right)-a_{i}\right)+\sum_{j=1}^{n}\left(\sigma\left(t_{j}\right)-t_{j}\right),
$$

if $A=\emptyset$, then $\mu_{\Delta}(A)=0$.

Definition 3.1 Let $\mathbb{T}$ be a time scale and $\Im_{T}$ be a field of subsets of $\mathbb{T}$. Suppose that $P_{\Delta}$ is a $\Delta$-measure defined on $\mathfrak{\Im}_{T}$. Then $P_{\Delta}$ is a probability measure if $P_{\Delta}(\mathbb{T})=1$. In this case, the triple $\left\{\mathbb{T}, \mathfrak{\Im}_{T}, P_{\Delta}\right\}$ is called a $\Delta$-probability space.

Definition 3.2 Let $\Omega_{T}$ be a sample space and $A \subset \Omega_{T}$, then

$$
P_{\Delta}(A)=\frac{\mu_{\Delta}(A)}{\mu_{\Delta}\left(\Omega_{T}\right)}
$$

is called $\Delta$-probability of $A$. Similarly,

$$
P_{\nabla}(A)=\frac{\mu_{\nabla}(A)}{\mu_{\nabla}\left(\Omega_{T}\right)}
$$

is called $\nabla$-probability of $A$.

Proposition 3.3 $P_{\Delta}$ and $P_{\nabla}$ are probability functions.

Proof Let $A \subset \Omega_{T}$. By using $0 \leq \mu_{\Delta}(A) \leq \mu_{\Delta}\left(\Omega_{T}\right)$, we get $0 \leq P_{\Delta}(A) \leq 1 . P_{\Delta}\left(\Omega_{T}\right)=1$ is clear by the definition. Let $A_{1}, A_{2}, \ldots$ be countable disjoint subsets of $\Omega_{T}$, then

$$
P_{\Delta}\left(\bigcup_{i=1}^{\infty} A\right)=\frac{\mu_{\Delta}\left(\bigcup_{i=1}^{\infty} A_{i}\right)}{\mu_{\Delta}\left(\Omega_{T}\right)}=\frac{\sum_{i=1}^{\infty} \mu_{\Delta}\left(A_{i}\right)}{\mu_{\Delta}\left(\Omega_{T}\right)}=\sum_{i=1}^{\infty} P_{\Delta}\left(A_{i}\right) .
$$

The proof of $P_{\nabla}$ is similar.

Example Let $\Omega_{T}=\{1,2,3,4, \ldots, n\}$ and $A=\{1,2,3, \ldots, m\}$, where $m<n$. Then

$$
P_{\Delta}(A)=\frac{\mu_{\Delta}(A)}{\mu_{\Delta}\left(\Omega_{T}\right)}=\frac{m}{n}
$$

which is equivalent to the counting probability. 
Proposition 3.4 For any $A, B \subset \Omega_{T}$, we have $P_{\Delta}(A) \leq P_{\Delta}(B)$ if $A \subset B$.

Proof Let $A \subset B \subset \mathbb{T}$. Then

$$
P_{\Delta}(A)=\frac{\mu_{\Delta}(A)}{\mu_{\Delta}\left(\Omega_{T}\right)} \leq \frac{\mu_{\Delta}(B)}{\mu_{\Delta}\left(\Omega_{T}\right)}=P_{\Delta}(B) .
$$

\section{Discrete random variables on time scales}

Definition 4.1 A random variable $X_{\mathbb{T}}$ is a real-valued function defined on $\mathfrak{\Im}_{T}$.

In this section we consider the binomial, Poisson, geometric, and negative binomial random variables on $\mathbb{T}=h \mathbb{N}$, where $h>0$.

\subsection{Binomial random variable on $h \mathbb{N}$}

Consider the time scale $\mathbb{T}=h \mathbb{N}, h>0$. Lets there are $n$ Bernoulli trials and but each Bernoulli trial has $h$ independent Bernoulli trials with probability of at least $k$ times success $p_{h}$ are performed independently, then $X_{\mathbb{T}}$, the number of trials for $x$ successes, is called the binomial random variable on the time scale with parameters $n, k$, and $p_{h}$. The probability function of this random variable is defined as follows:

$$
p(x)=P\left(X_{\mathbb{T}}=x_{t}\right)=\left(\begin{array}{c}
\frac{n}{\mu(x)} \\
x
\end{array}\right) p_{h}^{x} q_{h}^{\frac{n}{\mu(x)}-x},
$$

where $x_{t}=h x, \mu(x)$ is a grainness function, $p_{h}=\sum_{i=k}^{h}\left(\begin{array}{l}h \\ i\end{array}\right) p^{i} q^{h-i}$ and $q_{h}=1-p_{h}$ is called a binomial random variable on the time scale. Since $\mu(x)=x+h-x=h$, we take $h$ instead of $\mu(x)$.

Example Consider a jury trial in which it takes eight out of twelve juror groups to convict; that is, in order for the defendant to be convicted, at least eight of the juror groups must vote him guilty. Also, consider each group consists of three members. If at least two of three members vote that the defendant is guilty, then the decision of the group is guilty. If we assume that each juror group acts independently and each person makes the right decision with probability $\theta$, what is the probability that the jury renders a correct decision?

Let the defendant be innocent. The probability of the juror's right decision is

$$
p_{1}=\sum_{i=5}^{12}\left(\begin{array}{c}
12 \\
i
\end{array}\right) \theta_{h}^{i}\left(1-\theta_{h}\right)^{12-i}
$$

where $\theta_{h}=\left(\begin{array}{l}3 \\ 2\end{array}\right) \theta^{2}(1-\theta)+\left(\begin{array}{l}3 \\ 3\end{array}\right) \theta^{3}$.

Let the defendant be guilty. Then the probability of the juror's correct decision is

$$
p_{2}=\sum_{i=8}^{12}\left(\begin{array}{c}
12 \\
i
\end{array}\right) \theta_{h}^{i}\left(1-\theta_{h}\right)^{12-i}
$$

If $\alpha$ represents the probability that the defendant is guilty, then $\alpha p_{1}+(1-\alpha) p_{2}$ is the desired result.

While evaluating the expected value and the variance of the discrete random variables, we will make use of the following proposition. 
Proposition 4.2 If $X_{\mathbb{T}}$ is a discrete random variable that takes on one of the values $x_{i}, i \geq 1$, with the respective probabilities $p\left(x_{i}\right)$, then for any real-valued function $g$, $E[g(X)]=\sum_{i} g\left(x_{i}\right) p\left(x_{i}\right)$.

The expected value of a binomial random variable is given as follows:

$$
\begin{aligned}
E\left[X_{\mathbb{T}}^{s}\right] & =\sum_{i=0}^{\frac{n}{h}} i^{s} P\left(X_{\mathbb{T}}=i\right)=\sum_{i=0}^{\frac{n}{h}} i^{s}\left(\begin{array}{c}
\frac{n}{h} \\
i
\end{array}\right) p_{h}^{i} q_{h}^{\frac{n}{h}-i} \\
& =\sum_{i=1}^{\frac{n}{h}} i^{s}\left(\begin{array}{c}
\frac{n}{h} \\
i
\end{array}\right) p_{h}^{i} q_{h}^{\frac{n}{h}-i} \\
& =p_{h} \sum_{i=1}^{\frac{n}{h}} i\left(\begin{array}{c}
\frac{n}{h} \\
i
\end{array}\right) i^{s-1} p_{h}^{i-1} q_{h}^{\frac{n}{h}-i}=p_{h} \sum_{i=1}^{\frac{n}{h}} \frac{n}{h}\left(\begin{array}{c}
\frac{n}{h}-1 \\
i-1
\end{array}\right) i^{s-1} p_{h}^{i-1} q_{h}^{\frac{n}{h}-i} \\
& =\frac{n p_{h}}{h} \sum_{i=0}^{\frac{n}{h}-1}\left(\begin{array}{c}
\frac{n}{h}-1 \\
i
\end{array}\right)(i+1)^{s-1} p_{h}^{i} q_{h}^{\frac{n}{h}-1-i}=\frac{n p_{h}}{h} E\left[(Y+1)^{s-1}\right],
\end{aligned}
$$

where $Y$ is a random variable with parameters $\frac{n}{h}-1$ and $p_{h}$. If we set $s=1$, we get

$$
E\left[X_{\mathbb{T}}\right]=\frac{n p_{h}}{h}
$$

Remark 4.3 When we take $h=1$, then the time scale is a set of natural numbers and the expected value is as in the classical probability theory.

$$
\text { If } s=2 \text {, }
$$

$$
\begin{aligned}
E\left[X_{\mathbb{T}}^{2}\right] & =\frac{n p_{h}}{h} E[Y+1] \\
& =\frac{n p_{h}}{h}(E[Y]+1) \\
& =\frac{n p_{h}}{h}\left(\left(\frac{n}{h}-1\right) p_{h}+1\right) .
\end{aligned}
$$

So, the variance of a binomial random variable is

$$
\begin{aligned}
\operatorname{Var}\left(X_{\mathbb{T}}\right) & =E\left[X_{\mathbb{T}}^{2}\right]-E\left[X_{\mathbb{T}}\right]^{2} \\
& =\frac{n p_{h} q_{h}}{h} .
\end{aligned}
$$

Remark 4.4 When we take $h=1$, then the time scale is a set of natural numbers and the variance is as in the classical probability theory.

\subsection{Poisson random variable on $h \mathbb{N}$}

Definition 4.5 Let $\mathbb{T}=h \mathbb{N}$ be the time scale. A random variable $X_{\mathbb{T}}$ with possible values $0, h, 2 h, \ldots$ is called a Poisson random variable on the time scale with the parameter $\lambda>0$,

$$
p(t)=P\left(X_{\mathbb{T}}=t\right)=e^{-\lambda} \frac{\lambda^{\frac{t}{\mu(t)}}}{\mu(t)\left(\frac{t}{\mu(t)}\right) !} .
$$


Since

$$
\begin{aligned}
\int_{t \in \mathbb{T}} p(t) \Delta t & =\int_{0}^{h} p(t) \Delta t+\int_{h}^{2 h} p(t) \Delta t+\cdots \\
& =p(0) h+p(h) h+p(2 h) h+\cdots \\
& =h(p(0)+p(h)+p(2 h)+\cdots) \\
& =h \sum_{i=0}^{\infty} p(i h) \\
& =h \sum_{i=0}^{\infty} e^{-\lambda} \frac{\lambda^{\frac{i h}{\mu(t)}}}{h\left(\frac{i h}{\mu(t)}\right) !} \\
& =h \sum_{i=0}^{\infty} e^{-\lambda} \frac{\lambda^{\frac{i h}{h}}}{h\left(\frac{i h}{h}\right) !} \\
& =e^{-\lambda} \sum_{i=0}^{\infty} \frac{\lambda^{i}}{i !}=1,
\end{aligned}
$$

then $p(t)$ is a probability mass function.

The Poisson probability function is the limit of a binomial probability function, the expected value of a binomial random variable with parameters $\left(\frac{n}{h}, p_{h}\right)$ is $\frac{n}{h} p_{h}=\lambda$. It is reasonable to expect that the mean of the Poisson random variable with the parameter $\lambda$ is $\lambda$ as follows:

$$
\begin{aligned}
E\left[X_{\mathbb{T}}\right] & =\int_{t \in \mathbb{T}} t p(t) \Delta t=\int_{t \in \mathbb{T}} t e^{-\lambda} \frac{\lambda^{\frac{t}{h}}}{h\left(\frac{t}{h}\right) !} \Delta t \\
& =\frac{e^{-\lambda}}{h} \sum_{n=0}^{\infty} \int_{n h}^{\sigma(n h)} t \frac{\lambda^{\frac{t}{h}}}{\left(\frac{t}{h}\right) !} \Delta t=\frac{e^{-\lambda}}{h} \sum_{n=0}^{\infty} n h \frac{\lambda^{n}}{n !} \\
& =\lambda e^{-\lambda} \sum_{n=1}^{\infty} \frac{\lambda^{n-1}}{(n-1) !}=\lambda .
\end{aligned}
$$

For the variance of the Poisson random variable on $h \mathbb{N}$, we first compute

$$
\begin{aligned}
E\left[X_{\mathbb{T}}^{2}\right] & =\int_{t \in \mathbb{T}} t^{2} p(t) \Delta t \\
& =\int_{t \in \mathbb{T}} t^{2} e^{-\lambda} \frac{\lambda^{\frac{t}{\mu(t)}}}{\mu(t)\left(\frac{t}{\mu(t)}\right) !} \Delta t \\
& =\int_{t \in \mathbb{T}} t^{2} e^{-\lambda} \frac{\lambda^{\frac{t}{h}}}{h\left(\frac{t}{h}\right) !} \Delta t \\
& =e^{-\lambda} \lambda \int_{t \in \mathbb{T}} t \frac{\lambda^{\frac{t}{h}}-1}{\left(\frac{t}{h}-1\right) !} \Delta t \\
& =e^{-\lambda} \lambda \sum_{n=0}^{\infty} \int_{n h}^{\sigma(n h)} t \frac{\lambda \frac{t}{h}-1}{\left(\frac{t}{h}-1\right) !} \Delta t
\end{aligned}
$$




$$
\begin{aligned}
& =e^{-\lambda} \lambda\left(\int_{0}^{h} t \frac{\lambda^{\frac{t}{h}-1}}{\left(\frac{t}{h}-1\right) !} \Delta t+\int_{h}^{2 h} t \frac{\lambda^{\frac{t}{h}-1}}{\left(\frac{t}{h}-1\right) !} \Delta t+\cdots\right) \\
& =e^{-\lambda} \lambda\left(0 \frac{\lambda^{0}}{0 !}+1 \frac{\lambda^{1}}{1 !}+2 \frac{\lambda^{2}}{2 !}+\cdots\right) \\
& =e^{-\lambda} \lambda \sum_{k=0}^{\infty}(k+1) \frac{\lambda^{k}}{k !} \\
& =e^{-\lambda} \lambda\left(\sum_{k=0}^{\infty} k \frac{\lambda^{k}}{k !}+\sum_{k=0}^{\infty} \frac{\lambda^{k}}{k !}\right) \\
& =e^{-\lambda} \lambda\left(\lambda e^{\lambda}+e^{\lambda}\right)=\lambda(\lambda+1) .
\end{aligned}
$$

Therefore,

$$
\operatorname{Var}\left(X_{\mathbb{T}}\right)=E\left[X_{\mathbb{T}}^{2}\right]-E\left[X_{\mathbb{T}}\right]^{2}=\lambda
$$

Example An energy company produces batteries and sells five in a box. The probability that a battery is defective is 0.1. We assume if a box contains at least two defective batteries, then this box is also defective. Find the probability that a sample of ten boxes contains at most one defective.

By a binomial random variable, the desired probability is

$$
\left(\begin{array}{c}
10 \\
0
\end{array}\right) p_{h}^{0} q_{h}^{10}+\left(\begin{array}{c}
10 \\
1
\end{array}\right) p_{h} q_{h}^{9}
$$

where

$$
p_{h}=\sum_{i=2}^{5}\left(\begin{array}{l}
5 \\
i
\end{array}\right)(0.1)^{i}(0.9)^{5-i}
$$

and $q_{h}=1-p_{h}$. So, the desired probability is 0.806708 , whereas the Poisson approximation yields the value

$$
\sum_{i=0}^{1} e^{-10 p_{h}} \frac{\left(10 p_{h}\right)^{i}}{i !} \simeq 0.803535
$$

\subsection{Geometric random variable on $h \mathbb{N}$}

Suppose that independent trial groups, each having the probability $p_{h}, 0<p_{h}<1$, of being a success, are performed until a success occurs. If we let $X_{\mathbb{T}}$ equal the number of trials required, then we define

$$
P\left(X_{\mathbb{T}}=n\right)=\left(1-p_{h}\right)^{\frac{n}{h}-1} p_{h}, \quad n=h, 2 h, \ldots
$$

Equation (4.5) follows because in order for $X_{\mathbb{T}}$ to equal $\frac{n}{h}$, it is necessary and sufficient that the first $\frac{n}{h}-1$ trial groups are failures and the $\frac{n}{h}$ th trial group is a success. Equation (4.5) then follows, since the outcomes of the successive trial groups are assumed to be independent. 
Since

$$
\begin{aligned}
\sum_{n / h=1}^{\infty}\left(1-p_{h}\right)^{\frac{n}{h}-1} p_{h} & =p_{h} \sum_{n / h=1}^{\infty}\left(1-p_{h}\right)^{\frac{n}{h}-1} \\
& =p_{h} \frac{1}{1-\left(1-p_{h}\right)}=1,
\end{aligned}
$$

it follows that with probability one, a success group will eventually occur.

Definition 4.6 Any random variable $X_{\mathbb{T}}$ whose probability mass function is given by Equation (4.5) is said to be a geometric random variable with the parameter $p_{h}$.

By letting $\frac{n}{h}=k$, the expected value geometric random variable is given as follows:

$$
\begin{aligned}
E\left[X_{\mathbb{T}}\right] & =\sum_{k=1}^{\infty} k\left(1-p_{h}\right)^{k-1} p_{h} \\
& =p_{h} \frac{d}{d q_{h}}\left(\sum_{k=0}^{\infty} q_{h}^{k}\right) \\
& =p_{h} \frac{d}{d q_{h}}\left(\frac{1}{1-q_{h}}\right) \\
& =p_{h} \frac{1}{\left(1-q_{h}\right)^{2}}=\frac{1}{p_{h}} .
\end{aligned}
$$

To determine the variance of a geometric random variable, we first compute $E\left[X^{2}\right]$.

$$
\begin{aligned}
E\left[X^{2}\right] & =\sum_{k=1}^{\infty} k^{2}\left(1-p_{h}\right)^{k-1} p_{h}=p_{h} \sum_{k=1}^{\infty} k^{2} q_{h}^{k-1}=p_{h} \sum_{k=1}^{\infty} \frac{d}{d q_{h}}\left(k q_{h}^{k}\right) \\
& =p_{h} \frac{d}{d q_{h}}\left(\sum_{k=1}^{\infty} k q_{h}^{k}\right)=p_{h} \frac{d}{d q_{h}}\left(\frac{q_{h}}{1-q_{h}} E\left[X_{\mathbb{T}}\right]\right)=p_{h} \frac{d}{d q_{h}}\left(q_{h}\left(1-q_{h}\right)^{-2}\right) \\
& =p_{h}\left(\frac{1}{p_{h}^{2}}+\frac{2\left(1-p_{h}\right)}{p_{h}^{3}}\right)=\frac{2}{p_{h}^{2}}-\frac{1}{p_{h}} .
\end{aligned}
$$

Hence,

$$
\operatorname{Var}\left(X_{\mathbb{T}}\right)=E\left[X_{\mathbb{T}}^{2}\right]-\left(E\left[X_{\mathbb{T}}\right]\right)^{2}=\frac{1-p_{h}}{p_{h}^{2}}
$$

\subsection{Negative binomial random variable on $h \mathbb{N}$}

Negative binomial random variables on $h \mathbb{N}$ are generalizations of geometric random variables on $h \mathbb{N}$. Suppose that a sequence of Bernoulli independent trials, each trial is repeated $h$ Bernoulli trials with probability of at least k-times success $p_{h}$, are performed. Let $X_{\mathbb{T}}$ be the number of experiments until $i$ th success occurs, then it is called a negative binomial random variable if

$$
P\left(X_{\mathbb{T}}=n\right)=\left(\begin{array}{c}
\frac{n}{h}-1 \\
i-1
\end{array}\right) p_{h}^{i} q_{h}^{\frac{n}{h}-i}, \quad n=i h,(i+1) h,(i+2) h, \ldots
$$


The expected value and the variance of a negative binomial random variable

$$
\begin{aligned}
E\left[X_{\mathbb{T}}^{s}\right] & =\sum_{m=i}^{\infty} m^{s}\left(\begin{array}{c}
m-1 \\
i-1
\end{array}\right) p_{h}^{i}\left(1-p_{h}\right)^{m-i} \\
& =\frac{i}{p_{h}} \sum_{m=i}^{\infty} m^{s-1}\left(\begin{array}{c}
m \\
i
\end{array}\right) p_{h}^{i+1}\left(1-p_{h}\right)^{m-i} \\
& =\frac{i}{p_{h}} \sum_{m=i+1}^{\infty}(m-1)^{s-1}\left(\begin{array}{c}
m-1 \\
i
\end{array}\right) p_{h}^{i+1}\left(1-p_{h}\right)^{m-(i+1)} \\
& =\frac{i}{p_{h}} E\left[(Y-1)^{s-1}\right],
\end{aligned}
$$

where $m=\frac{n}{h}$, and $Y$ is a negative binomial random variable on $h \mathbb{N}$ with parameters $i+1$, $p_{h}$. We use the identity

$$
m\left(\begin{array}{c}
m-1 \\
i-1
\end{array}\right)=i\left(\begin{array}{c}
m \\
i
\end{array}\right)
$$

in the third line of the preceding equation. Setting $s=1$ in $E\left[X_{\mathbb{T}}^{s}\right]$, we get the expected value of a negative binomial random variable on $h \mathbb{N}$,

$$
E\left[X_{\mathbb{T}}\right]=\frac{i}{p_{h}}
$$

Setting $s=2$ in $E\left[X_{\mathbb{T}}^{s}\right]$ and using Equation (4.9) gives that

$$
E\left[X_{\mathbb{T}}^{2}\right]=\frac{i}{p_{h}} E[Y-1]=\frac{i}{p_{h}}\left(\frac{i+1}{p_{h}}-1\right) .
$$

Therefore,

$$
\begin{aligned}
\operatorname{Var}\left(X_{\mathbb{T}}\right) & =\frac{i}{p_{h}}\left(\frac{i+1}{p_{h}}-1\right)-\left(\frac{i}{p_{h}}\right)^{2} \\
& =\frac{i\left(1-p_{h}\right)}{p_{h}^{2}} .
\end{aligned}
$$

Example A student takes multiple choice exams which have five questions with three choices. The student is successful if he/she gives at least three correct answers in an exam. What is the probability of the third success of the student in the tenth exam by guessing?

Here $\mathbb{T}=5 \mathbb{N}$ and $n$, the number of questions on exams, is 50 . So, by formula (4.8), we have

$$
P\left(X_{\mathbb{T}}=10\right)=\left(\begin{array}{l}
9 \\
2
\end{array}\right) p_{h}^{3} q_{h}^{7} \simeq 0.0639
$$

where $p_{h}=\left(\begin{array}{c}5 \\ 3\end{array}\right)\left(\frac{1}{3}\right)^{3}\left(\frac{2}{3}\right)^{2}+\left(\begin{array}{l}5 \\ 4\end{array}\right)\left(\frac{1}{3}\right)^{4}\left(\frac{2}{3}\right)^{1}+\left(\begin{array}{c}5 \\ 5\end{array}\right)\left(\frac{1}{3}\right)^{5}\left(\frac{2}{3}\right)^{0}=\frac{17}{81}$ and $q_{h}=1-p_{h}=\frac{64}{81}$. 


\subsection{Uniform random variable on the time scale}

Let $\mathrm{T}=\left[t_{0}, t_{1}\right] \cup\left[t_{2}, t_{3}\right] \cup \cdots \cup\left[t_{n-1}, t_{n}\right]$, where $t_{0}=a$ and $t_{n}=b$ and $S_{R}=\left\{t_{1}, t_{3}, \ldots, t_{2 k+1}\right\}$ be the set of right scattered points. A uniform $\Delta$-probability function on $\mathrm{T}$ can be defined as follows:

$$
f_{\Delta}(t)= \begin{cases}\frac{1}{\mu_{\Delta}([a, b))}, & \text { if } t \in \mathrm{T} \\ 0, & \text { otherwise }\end{cases}
$$

Since this function satisfies the following condition:

$$
\begin{aligned}
\int_{-\infty}^{\infty} f_{\Delta}(t) \Delta(t) & =\int_{-\infty}^{a} f_{\Delta}(t) \Delta(x)+\int_{a}^{b} f_{\Delta}(t) \Delta(x)+\int_{b}^{\infty} f_{\Delta}(t) \Delta(x) \\
& =\int_{a}^{t_{1}} \frac{1}{\mu_{\Delta}([a, b))} \Delta(x)+\cdots+\int_{t_{n-1}}^{t_{n}} \frac{1}{\mu_{\Delta}([a, b))} \Delta(x) \\
& =\frac{1}{\mu_{\Delta}([a, b))} \sum_{i=1}^{n} \mu_{\Delta}\left(\left[t_{i-1}, t_{i}\right)\right) \\
& =\frac{b-a}{b-a}=1,
\end{aligned}
$$

this function is a probability function. Also, a uniform $\Delta$-probability distribution function on the time scale is defined as follows:

$$
F_{\Delta}(t)= \begin{cases}0, & t<a \text { or } t \in\left(t_{2 i+1}, \sigma\left(t_{2 i+1}\right)\right), \text { where } t_{2 i+1} \text { is right scattered, } \\ \frac{\mu_{\Delta}([a, t) \cap \Omega)}{\mu_{\Delta}([a, b))}, & \text { if } t \in \mathrm{T}, \\ 1, & t \geq b .\end{cases}
$$

This function satisfies all the properties of the distribution function

$$
\begin{aligned}
\lim _{t \rightarrow+\infty} F_{\Delta}(t) & =\lim _{t \rightarrow+\infty} \frac{\mu_{\Delta}([a, t) \cap \Omega)}{\mu_{\Delta}([a, b))_{\mathrm{T}}} \\
& =\frac{\mu_{\Delta}([a, b))_{\mathrm{T}}}{\mu_{\Delta}([a, b))_{\mathrm{T}}} \\
& =1, \\
\lim _{t \rightarrow-\infty} F_{\Delta}(t) & =\lim _{t \rightarrow-\infty} \frac{\mu_{\Delta}([a, t) \cap \Omega)}{b-a} \\
& =\lim _{t \rightarrow-\infty} \frac{\mu_{\Delta}(\{a\})_{\mathrm{T}}}{b-a} \\
& =\frac{\sigma(a)-a}{b-a}=0 .
\end{aligned}
$$

Remark 4.7 If we take the left closed and right open interval on our time scale $\mathrm{T}$ such that $A=\left[t_{i}, t_{i+1}\right)$, then the integral over this set $\int_{A} f_{\Delta}(t) \Delta(t)=\int_{t_{i}}^{t_{i+1}} f_{\Delta}(t) \Delta(t)=$ $\frac{1}{\mu_{\Delta}([b-a))} \mu_{\Delta}\left(\left[t_{i}, t_{i+1}\right)\right)=\frac{b-a}{b-a}=1$, and also, if we take right and left open intervals and since $a$ is right dense, then our result is the same $\int_{A} f_{\Delta}(t) \Delta(t)=\int_{t_{i}}^{t_{i+1}} f_{\Delta}(t) \Delta(t)+\int_{t_{i}}^{\sigma\left(t_{i+1}\right)} f_{\Delta}(t) \Delta(t)=$ $\frac{1}{\mu_{\Delta}((b-a))} \mu_{\Delta}\left(\left(t_{i}, t_{i+1}\right)\right)=\frac{b-\sigma(a)}{b-a}=1$. 


\section{Gaussian bell on time scales}

The continuous Gaussian bell $f(t)=e^{-\frac{t^{2}}{2}}$ satisfies the initial value problem

$$
f^{\prime}(t)=-t f(t), \quad f(0)=1
$$

The Gaussian bell is an even function, then the infinite time scale should be symmetric with respect to zero and contain zero. We denote the positive part of $\mathbb{T}$ by $\mathbb{T}_{+}$. So, the Gaussian bell $f_{\mathbb{T}}$ on the time scale should satisfy the following relation $\forall t \in \mathbb{T}_{+}$:

$$
f_{\mathbb{T}}^{\Delta}(t)=p(t) f(t), \quad f_{\mathbb{T}}(0)=1,
$$

where $p(t)$ must be $-t$ in the case $\mathbb{T}=\mathbb{R}$. Erbe and Peterson [7] defined the Gaussian bell on the time scale as follows.

Definition 5.1 On the time scale $\mathbb{T}$, we define the Gaussian bell $f_{\mathbb{T}}$ to be the unique solution of the initial value problem for all $t \in \mathbb{T}_{+}$

$$
f_{\mathbb{T}}^{\Delta}(t)=\ominus(t \odot 1) f(t), \quad f_{\mathbb{T}}(0)=1
$$

and $f_{\mathbb{T}}(-t)=f_{\mathbb{T}}(t)$

By using the definition of $\odot$ (circle dot) and $\ominus$ (circle minus) operations [3], if $\mu(t)>0$,

$$
\ominus(t \odot 1)=\frac{(1+\mu(t))^{-t}-1}{\mu(t)} .
$$

By the definition of $\Delta$ derivative, Equations (5.1) and (5.2), we get

$$
f_{\mathbb{T}}(\sigma(t))=(1+\mu(t))^{-t} f_{\mathbb{T}}(t), \quad \forall t \in \mathbb{T}_{+}, f_{\mathbb{T}}(0)=1
$$

Since $f_{\mathbb{T}}(t)$ satisfies the differential equation of the continuum Gaussian bell $f(t)$ at rightdense points $t \in \mathbb{T}_{+}$, thus it has a non-positive derivative at those points. We can conclude that $f_{\mathbb{T}}(t)$ is non-increasing on $\mathbb{T}_{+}$. On the discrete time scale $\mathbb{T}$, i.e., a time scale $\mathbb{T}$ containing no continuum intervals, we can write $f_{\mathbb{T}}$ as

$$
f_{\mathbb{T}}(t)=\prod_{x \in[0, t)}(1+\mu(x))^{-x}, \quad \forall t \in \mathbb{T}_{+}
$$

Example Consider $\mathbb{T}=h \mathbb{Z}, h>0$. So, $\mu(t)=h$, substituting $t=h n$, we get

$$
f_{\mathbb{T}}(h n)=\prod_{x \in[0, t)}(1+h)^{-x}=(1+h)^{-h \sum_{k=0}^{n-1} k}=(1+h)^{\frac{h n(1-n)}{2}}, \quad \forall n \in \mathbb{N}_{0},
$$

which implies $f_{\mathbb{T}}(t)=\left[(1+h)^{\frac{1}{h}}\right]^{\frac{-t(t-h)}{2}}, \forall t \in \mathbb{T}_{+}$.

For large $t, e^{\frac{t^{2}}{2}} \leq f_{\mathbb{T}}(t), f_{\mathbb{T}}(t)$ converges to the continuum Gaussian bell as $h \rightarrow 0 ; \forall t \in$ $[h n, h(n+1)], n \in \mathbb{N}_{0}$,

$$
\lim _{h \rightarrow 0} f_{\mathbb{T}}(h t)=\lim _{h \rightarrow 0}\left[(1+h)^{\frac{1}{h}}\right]^{-\frac{t h^{2}(t-1)}{2}}=e^{-\frac{t^{2}}{2}}, \quad \forall t \in \mathbb{R} .
$$


Example Let $\mathbb{T}=\bigcup_{n=0}^{\infty}[2 n, 2 n+1]$. Then the Gaussian bell on $\mathbb{T}$ is

$$
f_{\mathbb{T}}(t)=\left(\frac{e}{2}\right)^{n^{2}} e^{\frac{n-t^{2}}{2}}, \quad \forall t \in[2 n, 2 n+1]
$$

Mathematical induction is used for showing Equation (5.6).

In general, a probability distribution function and expected value of a random variable on a time scale can be defined as follows:

$$
\begin{aligned}
& F_{\Delta}(x)=\int_{-\infty}^{x} p(t) \Delta t, \\
& E(x)=\int_{-\infty}^{\infty} t p(t) \Delta t .
\end{aligned}
$$

By using an exponential function on time scales, we can define an exponential probability density function in a general case and we can define a moment generating function by using Laplace transformations on time scales. Then future works can be stochastic processes on time scales and stochastic dynamic equations.

\section{Competing interests}

The author declares that he has no competing interests.

\section{Acknowledgements}

I would like to thank TUBITAK and the referees for their support and their valuable comments.

Received: 14 August 2012 Accepted: 27 November 2012 Published: 10 December 2012

\section{References}

1. Hilger, S: Analysis on measure chains - a unified approach to continuous and discrete calculus. Results Math. 18, 18-56 (1990)

2. Bohner, M, Peterson, A: Dynamic Equations on Time Scales: an Introduction with Applications. Birkhauser, Boston (2001)

3. Bohner, M, Peterson, A: Advances in Dynamic Equations on Time Scales. Birkhauser, Boston (2004)

4. Agarwal, RP, Bohner, M, O’Regan, D, Peterson, A: Dynamic equations on time scales: a survey. J. Comput. Appl. Math. $141,1-26(2002)$

5. Agarwal, RP, Bohner, M, Rehak, P: Half-linear dynamic equations. In: Nonlinear Analysis and Applications. Kluwer Academic (to appear)

6. Neidhart, L: Integration on measure chains. In: Aulbach, B, Elaydi, S, Ladas, G (eds.) Conference Proceedings of the Sixth International Conference on Difference Equations. Taylor and Francis, Augsburg (2001)

7. Erbe, L, Peterson, A, Simon, M: Square integrability of Gaussian bells on time scales. Comput. Math. Appl. 4, 871-883 (2005)

8. Guseinov, GS: Integration on time scales. J. Math. Anal. Appl. 285(1), 107-127 (2003)

9. Guseinov, GS, Kaymakcalan, B: Basics of Riemann delta and nabla integration on time scales. J. Differ. Equ. Appl. 8(11), 1001-1017 (2002)

10. Cabada, A, Vivero, DR: Expression of Lebesgue Delta integral on timescales as a usual Lebesgue integral: application to the calculus of delta antiderivative. Math. Comput. Model. 43(1-2), 194-207 (2006)

11. Ufuktepe, U, Deniz, A: Lebesgue-Stieltjes measure on time scales. Turk. J. Math. 32, 1-8 (2009)

12. Rzezuchowski, T: A note on measure on time scales. Demonstr. Math. 38(1), 79-84 (2005)

13. Royden, HL: Real Analysis. Macmillan, New York (1988) 